\title{
Scleroderma with Acro-Osteolysis and Papular Mucinosis Resembling Multicentric Reticulohistiocytosis
}

\author{
João Dantas ${ }^{1}$, Isabela Silva de Oliveira², Emanuela Pimenta da Fonseca ${ }^{3,4}$, Mittermayer B Santiago ${ }^{1,5,6}$ \\ ${ }^{1}$ Hospital Universitário Professor Edgard Santos, Salvador, Bahia, Brazil \\ ${ }^{2}$ Universidade Federal da Bahia, Faculdade de Farmácia, Salvador, Bahia, Brazil \\ ${ }^{3}$ IBIS, Instituto Bahiano de Imunoterapia, Salvador, Bahia, Brazil \\ ${ }^{4}$ FTC, Faculdade de Tecnologia e Ciências, Salvador, Bahia, Brazil \\ ${ }^{5}$ Escola Bahiana de Medicina e Saúde Pública, Brotas, Salvador, Bahia, Brazil \\ ${ }^{6}$ Serviços Especializados em Reumatologia da Bahia, Salvador, Bahia, Brazil
}

Received: 10/02/2020

Accepted: $31 / 03 / 2020$

Published: $25 / 05 / 2020$

How to cite this article: Dantas J, Silva de Oliveira I, Pimenta da Fonseca E, Santiago SM. Scleroderma with acro-osteolysis and papular mucinosis resembling multicentric reticulohistiocytis. EJCRIM 2020;7: doi:10.12890/2020_001568.

Conflicts of Interests: The Authors declare that there are no competing interests.

Acknowledgements: M.B.S. is currently receiving a scholarship from Conselho Nacional de Desenvolvimento Científico e Tecnológico (CNPq). We would like to thank Editage (www.editage.com) for English language editing.

This article is licensed under a Commons Attribution Non-Commercial 4.0 License

\section{ABSTRACT}

Objectives: We describe a case of systemic sclerosis (SS) with acro-osteolysis associated with cutaneous mucinosis, usually characterized by mucin deposition in the skin. The main differential diagnosis was multicentric reticulohistiocytosis due to the presentation of papulonodular skin lesions.

Materials and methods: A physical examination, imaging studies and laboratory tests were performed.

Results: Distal bone resorption was evident on plain radiographs, and skin biopsy confirmed mucinosis. The SS diagnosis was based on the clinical features, high levels of antinucleolar antibodies and typical nailfold capillaroscopy findings.

Conclusion: To the best of our knowledge, this is the first description of cutaneous mucinosis accompanying SS with acro-osteolysis.

\section{LEARNING POINTS}

- Cutaneous mucinosis is rarely associated with systemic sclerosis.

- Systemic sclerosis with cutaneous mucinosis may resemble multicentric reticulohistiocytosis.

\section{KEYWORDS}

Systemic sclerosis, cutaneous mucinosis, acro-osteolysis, multicentric reticulohistiocytosis

\section{INTRODUCTION}

Cutaneous mucinosis (CM) refers to a variety of skin conditions where mucin deposition is a hallmark. This particular feature has been described in some cases of systemic lupus erythematosus (SLE) ${ }^{[1]}$ and only rarely in other rheumatic diseases such as localized scleroderma and systemic sclerosis (SS) ${ }^{[2]}$. To the best of our knowledge, this is the first description of the association of SS with acro-osteolysis and papular mucinosis (PM). The main differential diagnosis was multicentric reticulohistiocytosis. 


\section{CASE DESCRIPTION}

A 60-year-old Brazilian woman presented for the last 6 years with cutaneous findings consisting of diffuse skin thickening in the trunk, forearms and hands, which was associated with asymptomatic clusters of firm, flesh-coloured papular and nodular lesions, predominantly on the hands, forearms and the extensor surface of the elbows. The papules were non-pruritic, with some coalescent, and had no inflammatory appearance, thus exhibiting a slow progression over time (Figs. 1A and 1B). She complained of fatigue, mild arthralgia, mostly in the hands and wrists, and mild dysphagia. She also demonstrated Raynaud's phenomenon solely in her hands. Besides the skin features, musculoskeletal examination revealed abnormalities in the distal interphalangeal (DIP) joints of the hands, resembling a clubbing pattern sparing the left fourth finger (Fig. 1A).

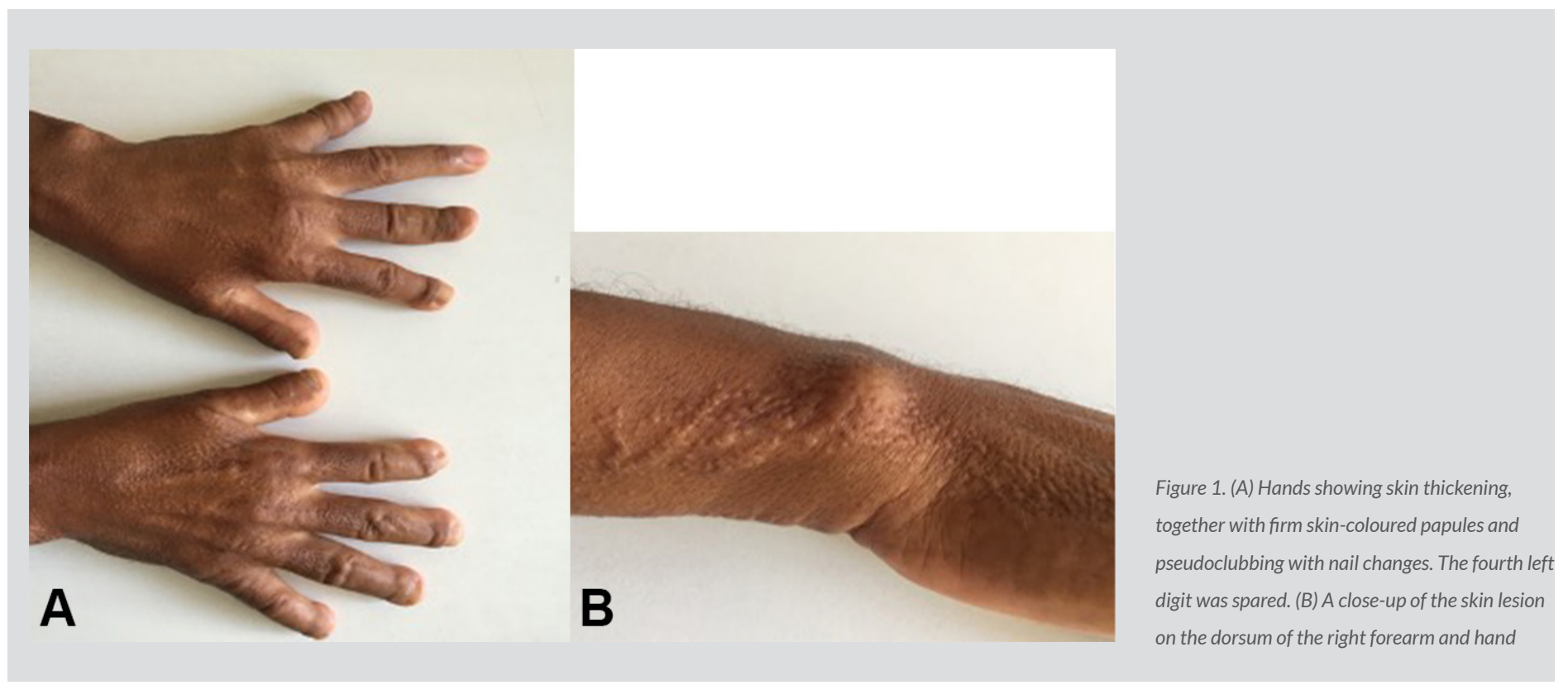

Some of the DIP joints were extremely loose and displayed subluxation. The rest of the physical examination was unremarkable. Laboratory work-up showed normal kidney and hepatic function as well as a normal white blood cell count, while haemoglobin and platelets, acute phase reactants, and complement and serum protein levels were within the normal ranges. Investigation with respect to viral infections showed negative results for HBV, HCV and HIV. She had a positive antinuclear antibody (ANA) test, 1:640 with both fine speckled nuclear and nucleolar patterns. Testing for serum anti-Scl-70 (anti-topoisomerase I) was negative. Plain radiographs of both hands showed mild marginal erosions in some of the metacarpophalangeal joints of both hands, which were most evident in the second right finger as well as in the proximal interphalangeal joints (Fig. 2). In the DIP joints, images showed significant bone resorption, shortening of the fingers and the third left finger with a pencil-like appearance, suggesting advanced phalanx resorption known as acro-osteolysis. The distal bone in the fourth ipsilateral finger showed no abnormalities, and thus was spared. Nailfold capillaroscopy showed significant pericapillary oedema, minor disorganization of the capillary architecture, extensive and tortuous capillary ectasia, some giant capillaries and mild neoangiogenesis. The number of capillaries was reduced with irregular blood flow. The final description was compatible with an active pattern of scleroderma. The skin biopsy revealed dermis with intense collagen infiltration and mucin deposition as evidenced by Alcian blue staining (Fig. 3), suggestive of scleroderma in addition to mucinosis.

\section{DISCUSSION}

The skin findings for our patient suggested a diagnosis of diffuse scleroderma in the first instance, mainly because of the easily noticeable skin thickening. However, papular and nodular skin lesions are not described within the spectrum of scleroderma itself; hence, the need to establish a thorough differential diagnosis. In this case, this included multicentric reticulohistiocytosis, a type of non-Langerhans cell histiocytosis, particularly due to the presence of abnormalities in the DIP joints. However, the histopathological features found in our patient excluded this hypothesis. Moreover, the presence of acro-osteolysis leading to a pseudoclubbing pattern of the fingers is not rare in $\mathrm{SS}^{[3]}$. This was more likely since the ANA test was positive with a nucleolar pattern (although serum was negative for anti-Scl-70 antibodies) and the nailfold capillaroscopy findings were suggestive of SS based on previously described criteria ${ }^{[3]}$. 

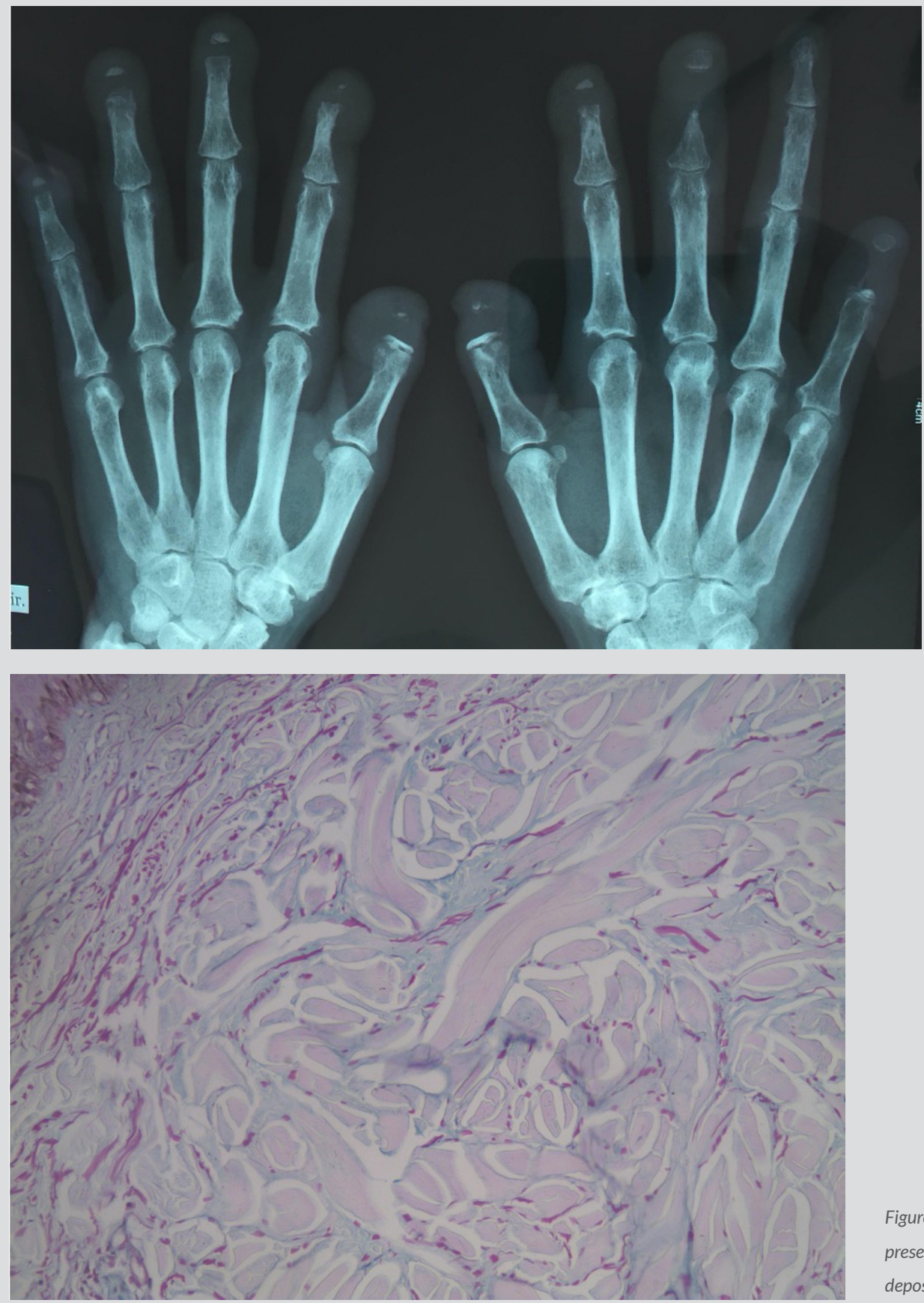

Figure 2. Resorption of distal phalanges and mild marginal erosions. Pencil-like appearance of the left third finger with apparent sparing of the ipsilateral fourth finger
Figure 3. Skin biopsy with Alcian blue staining, demonstrating the presence of mucin deposition in the context of intense collagen deposition

CM involves a focal or diffuse deposition of mucin in the interstitial tissue of the dermis, which can be a primary phenomenon or observed in association with connective tissue disorders such as SLE, localized scleroderma or SS ${ }^{11,2,4]}$. The pathogenesis of CM includes the overproduction of mucin, specifically hyaluronic acid, by pathologic fibroblasts. The pathogenetic mechanisms, although not completely understood, involve the participation of some cytokines. The main cytokine involved is transforming growth factor $\beta$ (TGF- $\beta$ ), which is able to stimulate fibroblasts to produce extracellular matrix components, such as collagen, fibronectin and glycosaminoglycans ${ }^{[5]}$. TGF- $\beta$ also reduces fibroblast apoptosis.

Currently, there is no consistently effective therapy for the treatment of CM, although systemic and locally administered glucocorticoids, antimalarials and injections of hyaluronidase have been used for this purpose in the past. Our patient has only been treated for the symptoms of oesophageal dysmotility, with no apparent progression of the disease. 


\section{REFERENCES}

1. Rongioletti F, Parodi A, Rebora A. Papular and nodular mucinosis as a sign of lupus erythematosus. Dermatologica 1990;180:221-223.

2. Rongioletti F, Gambini C, Micalazzi C, Pastorino A, Rebora A. Mucin deposits in morphea and systemic scleroderma. Dermatology 1994;189:157-158.

3. Santiago MB, Lima I, Feitosa AC, Braz AS, Miranda LG. Pseudoclubbing: is it different from clubbing? Semin Arthritis Rheum 2009;38:452-457.

4. Sawada Y, Seishima M, Funabashi M, Noda T, Maeda M, Kitajima Y. Papular mucinosis associated with scleroderma. Eur J Dermatol 1998;8(7):497-500.

5. Buckingham RB, Prince RK, Rodnan GP. Progressive systemic sclerosis dermal fibroblasts synthesize increased amounts of glycosaminoglycans. J Lab Clin Med 1983;101:659669. 